OPEN ACCESS

Edited by:

Paolo Peretto,

University of Turin, Italy

Reviewed by:

Martine Migaud,

Institut National de la Recherche Agronomique, Centre National de la Recherche Scientifique UMR7247,

France

Martin Holzenberger, Institut National de la Santé et de la

Recherche Médicale, France

*Correspondence:

María de los Angeles García-Robles mgarcia@udec.c

Specialty section

This article was submitted to Neurogenesis,

a section of the journal

Frontiers in Neuroscience

Received: 05 February 2017

Accepted: 21 March 2017

Published: 05 April 2017

Citation:

Recabal A, Caprile T and

García-Robles MA (2017)

Hypothalamic Neurogenesis as an Adaptive Metabolic Mechanism.

Front. Neurosci. 11:190.

doi: 10.3389/fnins.2017.00190

\section{Hypothalamic Neurogenesis as an Adaptive Metabolic Mechanism}

\author{
Antonia Recabal ${ }^{1,2}$, Teresa Caprile ${ }^{2}$ and María de los Angeles García-Robles ${ }^{1 *}$ \\ ${ }^{1}$ Laboratorio de Biología Celular, Departamento de Biología Celular, Facultad de Ciencias Biológicas, Universidad de \\ Concepción, Concepción, Chile, ${ }^{2}$ Laboratorio de Guía Axonal, Departamento de Biología Celular, Facultad de Ciencias \\ Biológicas, Universidad de Concepción, Concepción, Chile
}

In the adult brain, well-characterized neurogenic niches are located in the subventricular zone (SVZ) of the lateral ventricles and in the subgranular zone (SGZ) of the hippocampus. In both regions, neural precursor cells (NPCs) share markers of embryonic radial glia and astroglial cells, and in vitro clonal expansion of these cells leads to neurosphere formation. It has also been more recently demonstrated that neurogenesis occurs in the adult hypothalamus, a brain structure that integrates peripheral signals to control energy balance and dietary intake. The NPCs of this region, termed tanycytes, are ependymalglial cells, which comprise the walls of the infundibular recess of the third ventricle and contact the median eminence. Thus, tanycytes are in a privileged position to detect hormonal, nutritional and mitogenic signals. Recent studies reveal that in response to nutritional signals, tanycytes are capable of differentiating into orexigenic or anorexigenic neurons, suggesting that these cells are crucial for control of feeding behavior. In this review, we discuss evidence, which suggests that hypothalamic neurogenesis may act as an additional adaptive mechanism in order to respond to changes in diet.

Keywords: tanycytes, hypothalamus, glucosensing, feeding behavior, neurogenesis

\section{INTRODUCTION}

Postnatal neurogenesis corresponds to the series of events that lead to the production of new neurons in the adult brain, from precursor cell division to the survival and functional integration of newly differentiated neurons (Lledo et al., 2006). In the adult brain under normal conditions, wellcharacterized niches are restricted to the subventricular zone (SVZ) of lateral ventricles (Doetsch et al., 1999) and the hippocampal subgranular zone (SZG; Altman and Das, 1965). Experiments also suggest the existence of constitutive neurogenesis in close proximity to circumventricular organs (Hourai and Miyata, 2013).

Neurogenic cells present in the SVZ and SGZ share the following features: (i) astroglial marker expression, including glial fibrillary acidic protein (GFAP) and GLutamate ASpartate Transporter (GLAST) (Platel et al., 2009); (ii) stem cell marker expression, such as nestin and SOX2 (Imayoshi et al., 2008); and (iii) in vitro clonal expansion resulting in neurosphere formation (Lledo et al., 2006; Kriegstein and Alvarez-Buylla, 2009).

Neurogenesis also occurs in the adult hypothalamus (Evans et al., 2002; Cheng, 2013), a brain structure located at the base of the diencephalon, close to the third ventricle (3V) and in close contact to the median eminence (ME), a circumventricular organ. The present review will discuss the cell origin of the hypothalamic newborn neurons and its physiological role in the energy balance. 


\section{HYPOTHALAMUS AND ENERGY BALANCE}

The hypothalamus is the main regulator of energy balance and instinctive behaviors, including food intake. In the hypothalamus, the arcuate nucleus (AN) is composed of clustered neuronal populations that inhibit or initiate food intake through the release of anorexigenic or orexigenic peptides, respectively (Schwartz et al., 2000). There is great interest in understanding the precise molecular and cellular mechanisms that control glucosensing, especially given that diabetes and obesity may be induced by a dysregulation in this process (Elizondo-Vega et al., 2016).

Radial glial-like tanycytes surround the lateral walls of the infundibular recess. Their apical poles contact the cerebrospinal fluid (CSF), and the basal extensions project into the AN (Flament-Durand and Brion, 1985). Tanycytes are classified into four main groups on the basis of differences in their localization and gene expression: $\alpha 1, \alpha 2$ (Robins et al., 2013), $\beta 1$, and $\beta 2$ (Rodríguez et al., 2005). $\alpha 2$ and $\beta 1$ tanycytes are located in the lateral walls of the $3 \mathrm{~V}$ and contact anorexigenic and orexigenic neurons through their extensive processes. $\beta 2$-tanycytes cover the floor of the $3 \mathrm{~V}$ and present tight junctions that form the CSF-ME barrier and extend their projections inside the ME (Figure 1); their tight junctions and cellular contacts can change, depending on the metabolic state of the organism (Langlet et al., 2013).

It has been postulated that tanycytes function as neuromodulating cells, since they regulate the availability of hormones, such as leptin (Balland et al., 2014) and ghrelin (Collden et al., 2015), from peripheral tissues to neurons of the AN. They also express the molecular machinery that permits glucose sensing (García et al., 2003; Cortés-Campos et al., 2011; Orellana et al., 2012) and transmit signals to AN neurons (Elizondo-Vega et al., 2016). Due to their privileged position in the hypothalamus, tanycytes can also detect mitogenic and neurodifferentiating signals present in the peripheral blood or in the CSF (Robins et al., 2013; Chaker et al., 2016).

\section{TANYCYTES AS HYPOTHALAMIC NEURAL PRECURSOR CELLS (NPCS)}

Tanycytes have been suggested as possible hypothalamic precursors since they share the characteristics of the neuronal precursors of the SVZ and SGZ. Specifically, they express GFAP (Haan et al., 2013), GLAST (Robins et al., 2013), nestin and SOX2 (Lee et al., 2012; Li et al., 2012). Tanycytes also express the multipotent cell markers, UGS148 (Ma et al., 2015)

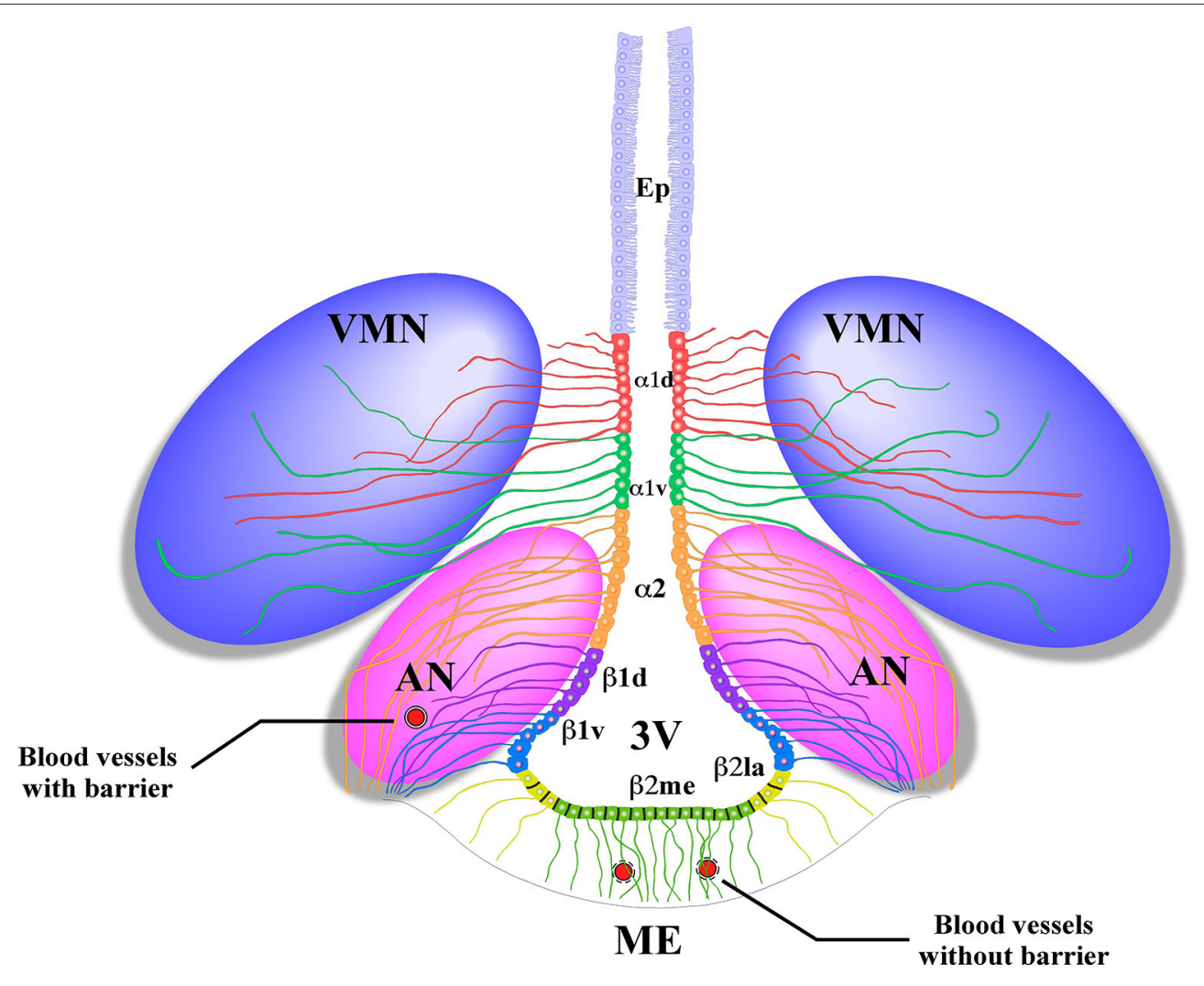

FIGURE 1 | Schematic representation of the basal hypothalamus. Ciliated ependymocytes (Ep) line the dorsal wall of the $3 \mathrm{~V}$. The $\alpha 1 \mathrm{~d}$-tanycytes ( $\alpha 1 \mathrm{~d})$ and $\alpha 1 \mathrm{v}$-tanycytes $(\alpha 1 \mathrm{v})$ have long projections that make contact with the neurons of the VMN. The $\alpha 2$-tancycytes $(\alpha 2) \beta 1 \mathrm{~d}$-tanycytes $(\beta 1 \mathrm{~d})$ and $\beta 1 \mathrm{~V}$-tanycytes $(\beta 1 \mathrm{v})$ make projections to the AN, making contact with orexigenic and anorexigenic neurons and blood vessels. In the floor of the $3 \mathrm{~V}$, the $\beta 2$ lateral-tanycytes ( $\beta 2 \mathrm{la}$ ) and $\beta 2$ medial-tanycytes ( $\beta 2 \mathrm{me}$ ) are joined by tight junctions forming part of the median eminence (ME)-cerebrospinal fluid (CSF) barrier, and their projections make contact with the fenestrated blood vessels of the ME. Redrawn from a previously published (Elizondo-Vega et al., 2015). 


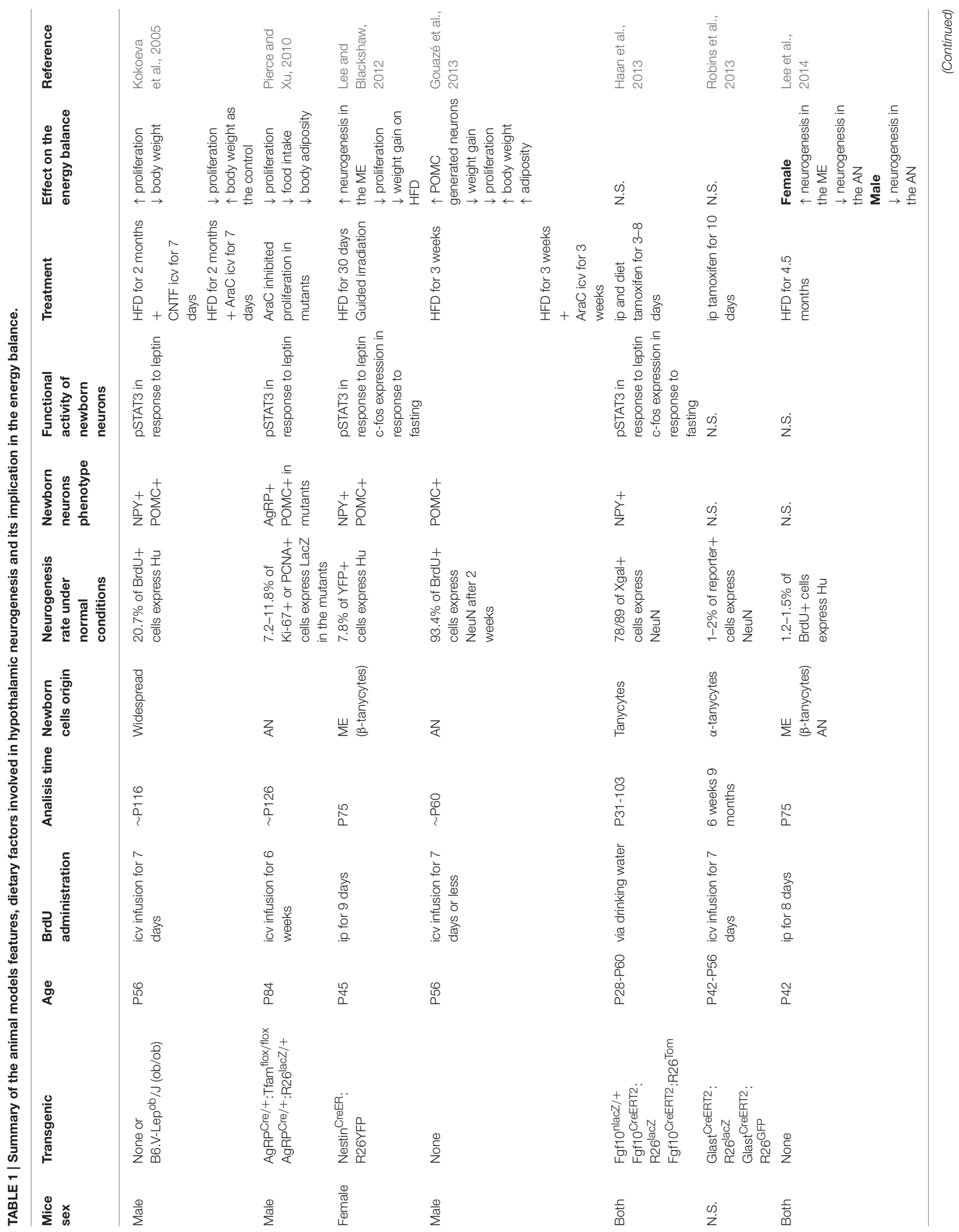




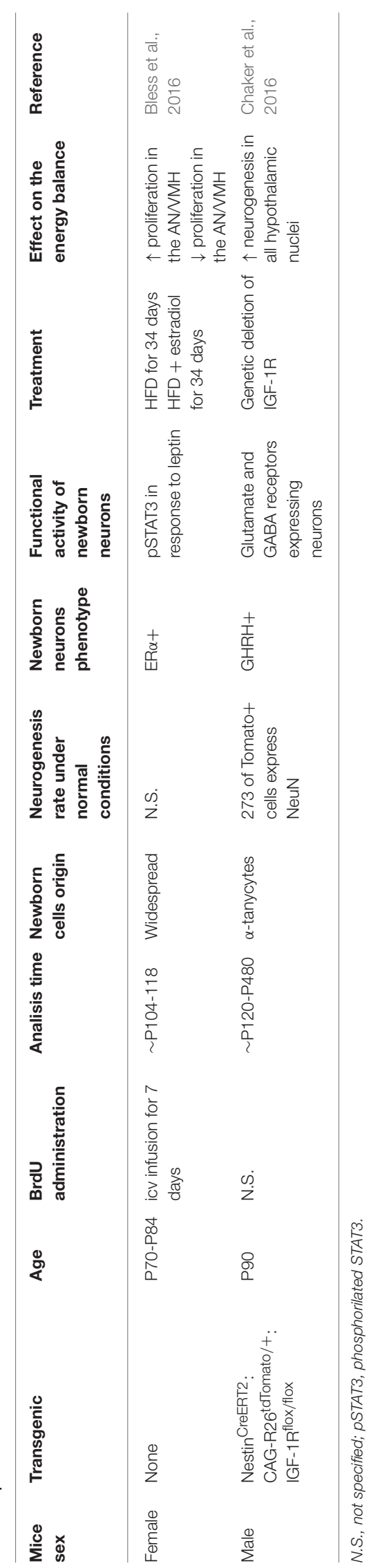

and FGF10 (Haan et al., 2013), and are capable of forming neurospheres (Robins et al., 2013). Different populations of tanycytes have different neurosphere-forming capabilities; dorsal $\alpha 2$ tanycytes actively proliferate and form new neurospheres after cell isolation, while $\beta$ tanycytes do not form neurospheres (Robins et al., 2013).

In lineage-tracing experiments using a Cre/lox system in which recombinase were expressed in tanycytes under the control of promoters, such as nestin (Lee and Blackshaw, 2012), glast (Robins et al., 2013), fgf10 (Haan et al., 2013), and prss56 (Jourdon et al., 2015), their role as neural stem cells was confirmed. In these transgenic mice, the constitutive expression of a reporter gene facilitates tanycyte tracking over time. The use of transgenic Cre/lox together with the intraperitoneal (ip) or intracerebroventricular (icv) delivery of BrdU has shown the selfrenewal of tanycytes and their differentiation into other cell types, including mature neurons that respond to peripheral signals (Table 1).

After successive divisions, hypothalamic progenitors begin to express proteins characteristic of migrating, immature neurons, including neural cell adhesion molecule with polysialic acid modification (PSA-NCAM) (Bonfanti et al., 1992); the microtubule binding protein, doublecortin (DCX), has also been found in human hypothalamic slices (Batailler et al., 2014). Some of the neuroblasts that originated from tanycytes are able to migrate to the $\mathrm{AN}$, differentiate to an orexigenic or anorexigenic neurons, and respond to peripheral signals, such as leptin (Kokoeva et al., 2005; Lee and Blackshaw, 2012). This suggests that the rate of neuronal renewal is not merely restorative; it consists of an adaptive mechanism in response to metabolic changes imposed by the environment and/or the internal state of the organism (Lledo et al., 2006).

\section{HYPOTHALAMIC NEUROGENESIS AS AN ADAPTIVE METABOLIC MECHANISM}

The normal proportion of newborn neurons among newborn cells in the adult rodent hypothalamus is lower (1-37\%; Migaud et al., 2010) than in the SGZ and SVZ (70-100\%; (Lledo et al., 2006)), but its rate increase after icv administration of ciliary neurotrophic factor (CNTF) (Kokoeva et al., 2005), insulin-like growth factor (IGF) (Pérez-Martín et al., 2010), brain derived neurotrophic factor (BNDF) (Pencea et al., 2001), and fibroblast growth factor 2 (FGF2) (Xu et al., 2005; Robins et al., 2013). These factors enhance proliferation of cells that are in proximity of the $3 \mathrm{~V}$, both into the parenchyma or ventricular zone. Specifically, it has been shown that FGF2 (Robins et al., 2013) and IGF-1R (Chaker et al., 2016) are directly involved in the regulation of $\alpha$-tanycyte proliferation, whereas circulating lipids promote $\beta$ tanycyte proliferation in female rats. The type of tanycyte that act as NPC may seem controversial, but the source of the triggering elements and the sex of the model studied must be considered (Lee et al., 2014).

Changes in metabolic conditions can also modify the proliferation of hypothalamic neuronal precursors (Table 1), including high temperatures (Matsuzaki et al., 2009), physical 
activity (Niwa et al., 2016), and a high fat diet (HFD) (Kokoeva et al., 2005; Lee and Blackshaw, 2012; Gouazé et al., 2013; Nascimento et al., 2016). During prenatal neurogenesis, in utero exposure to a HFD stimulates the production of orexigenic hypothalamic neurons (Chang et al., 2008), which causes changes in behavior and physiological conditions that extend into adulthood as demonstrated by the increased body weight and caloric intake observed in P70 (Chang et al., 2008).

Other experiments using adult rodents under a HFD have shown the following.

i. Anorexigenic neurogenesis is accelerated, preventing the weight gain and fat mass induced by the change in diet (Gouazé et al., 2013). Dietary or direct 3V injection of polyunsaturated fatty acids, such as docosahexaenoic acid (DHA), increases the generation of pro-opiomelanocortin (POMC)-expressing neurons, possibly through the interaction of GPR40 fatty acids receptors (Nascimento et al., 2016). Female but not male mice consuming a HFD had increased cell proliferation that was attenuated by estradiol in the AN (Bless et al., 2016).

ii. Chronic exposure to a HFD leads to the loss of mature hypothalamic orexigenic and anorexigenic neurons (Moraes et al., 2009), as well as loss of hypothalamic neuronal precursors (Li et al., 2012). The inflammatory microenvironment activated in preobesity and prediabetes impairs the survival of hypothalamic neuronal progenitors upon activation of I $\kappa$ B kinase B/nuclear $\kappa$ B factor (Li et al., 2012).

iii. Simultaneous icv infusion of cytosine- $\beta$-Darabinofuranoside (AraC) is sufficient to cause an exaggerated increase in body weight (Kokoeva et al., 2005; Gouazé et al., 2013), implying that hypothalamic neurogenesis may restore energy balance. In contrast, inhibition of mitosis in the ventromedial hypothalamus of female mice increases energy expenditure and diminishes body weight, suggesting that neuronal differentiation due to a HFD promotes energy storage (Lee and Blackshaw, 2012; Lee et al., 2014).

iv. Transgenic mice that have a progressive degeneration of AgRP orexigenic neurons due the genetic deletion of the mitochondrial transcription factor A (Tfam) originate a new

\section{REFERENCES}

Altman, J., and Das, G. D. (1965). Autoradiographic and histological evidence of postnatal hippocampal neurogenesis in rats. J. Comp. Neurol. 124, 319-335. doi: 10.1002/cne.901240303

Balland, E., Dam, J., Langlet, F., Caron, E., Steculorum, S., Messina, A., et al. (2014). Hypothalamic tanycytes are an ERK-gated conduit for leptin into the brain. Cell Metab. 19, 293-301. doi: 10.1016/j.cmet.2013. 12.015

Batailler, M., Droguerre, M., Baroncini, M., Fontaine, C., Prevot, V., and Migaud, M. (2014). DCX-expressing cells in the vicinity of the hypothalamic neurogenic niche: a comparative study between mouse, sheep, and human tissues. J. Comp. Neurol. 522, 1966-1985. doi: 10.1002/cne.23514

Bless, E. P., Yang, J., Acharya, K. D., Nettles, S. A., Vassoler, F. M., Byrnes, E. M., et al. (2016). Adult neurogenesis in the female mouse hypothalamus: estradiol and high-fat diet alter the generation of newborn subset of AgRP neurons, a fact that explains why mutant mice do not exhibit decreased body weight in response to neurodegeneration (Pierce and $\mathrm{Xu}, 2010$ ).

It should be noted that experiments differ in the promoter used, the sex and age of the animals and the chase term (Table 1). The added neurons may play a different role in the regulation of dietary intake and body weight depending on the hypothalamic region they established and the factors mentioned (Lee et al., 2014).

\section{CONCLUSION}

Hypothalamic neurogenesis can be stimulated by intrinsic factors, such as CSF-derived mitogenic molecules or peripheral factors that cross the ME. Additionally, this event can be promoted by environmental changes in the diet (e.g., HFD and calorie restriction) or neurodegeneration (e.g., genetically induced death of AgRP neurons). Both conditions promote the generation of new neurons as part of a response to restore energy balance prior to the development of metabolic diseases that prevent the generation and proper development of hypothalamic NSCs. The specific mechanisms that link changes in the diet with the proliferation of tanycytes remain unknown, but they may involve their well-known nutrient chemosensitive machinery and/or their metabolic coupling with $\mathrm{AN}$ neurons that control appetite.

\section{AUTHOR CONTRIBUTIONS}

AR conceived the review focus, conducted the literature review, and summarized and finalized the manuscript. TC and MG reviewed the literature, wrote the first draft, and finalized the manuscript. All authors approved final version of manuscript.

\section{FUNDING}

This study was funded by the National Fund for Scientific and Technological Development (FONDECYT number 1140677).

neurons expressing estrogen receptor $\alpha$. eNeuro 3:ENEURO.0027-16.2016. doi: 10.1523/ENEURO.0027-16.2016

Bonfanti, L., Olive, S., Poulain, D. A., and Theodosis, D. T. (1992). Mapping of the distribution of polysialylated neural cell adhesion molecule throughout the central nervous system of the adult rat: an immunohistochemical study. Neuroscience 49, 419-436. doi: 10.1016/0306-4522(92)90107-D

Chaker, Z., George, C., Petrovska, M., Caron, J. B., Lacube, P., Caillé, I., et al. (2016). Hypothalamic neurogenesis persists in the aging brain and is controlled by energy-sensing IGF-I pathway. Neurobiol Aging 41, 64-72. doi: 10.1016/j.neurobiolaging.2016.02.008

Chang, G. Q., Gaysinskaya, V., Karatayev, O., and Leibowitz, S. F. (2008). Maternal high-fat diet and fetal programming: increased proliferation of hypothalamic peptide-producing neurons that increase risk for overeating and obesity. $J$. Neurosci. 28, 12107-12119. doi: 10.1523/JNEUROSCI.2642-08.2008

Cheng, M. F. (2013). Hypothalamic neurogenesis in the adult brain. Front. Neuroendocrinol. 34, 167-178. doi: 10.1016/j.yfrne.2013.05.001 
Collden, G., Balland, E., Parkash, J., Caron, E., Langlet, F., Prevot, V., et al. (2015). Neonatal overnutrition causes early alterations in the central response to peripheral ghrelin. Mol. Metab. 4, 15-24. doi: 10.1016/j.molmet.2014.10.003

Cortés-Campos, C., Elizondo, R., Llanos, P., Uranga, R. M., Nualart, F., and García, M. A. (2011). MCT expression and lactate influx/efflux in tanycytes involved in glia-neuron metabolic interaction. PLoS ONE 6:e16411. doi: 10.1371/journal.pone.0016411

Doetsch, F., Caillé, I., Lim, D. A., García-Verdugo, J. M., and Alvarez-Buylla, A. (1999). Subventricular zone astrocytes are neural stem cells in the adult mammalian brain. Cell 97, 703-716. doi: 10.1016/S0092-8674(00)80783-7

Elizondo-Vega, R., Cortés-Campos, C., Barahona, M. J., Carril, C., Ordenes, P., Salgado, M., et al. (2016). Inhibition of hypothalamic MCT1 expression increases food intake and alters orexigenic and anorexigenic neuropeptide expression. Sci. Rep. 6:33606. doi: 10.1038/srep33606

Elizondo-Vega, R., Cortes-Campos, C., Barahona, M. J., Oyarce, K. A., Carril, C. A., and Garcia-Robles, M. A. (2015). The role of tanycytes in hypothalamic glucosensing. J. Cell. Mol. Med. 19, 1471-1482. doi: 10.1111/jcmm.12590

Evans, J., Sumners, C., Moore, J., Huentelman, M. J., Deng, J., Gelband, C. H., et al. (2002). Characterization of mitotic neurons derived from adult rat hypothalamus and brain stem. J. Neurophysiol. 87, 1076-1085. doi: 10.1152/jn.00088.2001

Flament-Durand, J., and Brion, J. P. (1985). Tanycytes: morphology and functions: a review. Int. Rev. Cytol. 96, 121-155. doi: 10.1016/S0074-7696(08)60596-3

García, M. A., Millán, C., Balmaceda-Aguilera, C., Castro, T., Pastor, P., Montecinos, H., et al. (2003). Hypothalamic ependymal-glial cells express the glucose transporter GLUT2, a protein involved in glucose sensing. J. Neurochem. 86, 709-724. doi: 10.1046/j.1471-4159.2003.01892.x

Gouazé, A., Brenachot, X., Rigault, C., Krezymon, A., Rauch, C., Nédélec, E., et al. (2013). Cerebral cell renewal in adult mice controls the onset of obesity. PLoS ONE 8:e72029. doi: 10.1371/journal.pone.0072029

Haan, N., Goodman, T., Najdi-Samiei, A., Stratford, C. M., Rice, R., El Agha, E., et al. (2013). Fgf10-expressing tanycytes add new neurons to the appetite/energy-balance regulating centers of the postnatal and adult hypothalamus. J. Neurosci. 33, 6170-6180. doi: 10.1523/JNEUROSCI.2437-12.2013

Hourai, A., and Miyata, S. (2013). Neurogenesis in the circumventricular organs of adult mouse brains. J. Neurosci. Res. 91, 757-770. doi: 10.1002/jnr.23206

Imayoshi, I., Sakamoto, M., Ohtsuka, T., Takao, K., Miyakawa, T., Yamaguchi, M., et al. (2008). Roles of continuous neurogenesis in the structural and functional integrity of the adult forebrain. Nat. Neurosci. 11, 1153-1161. doi: $10.1038 / \mathrm{nn} .2185$

Jourdon, A., Gresset, A., Spassky, N., Charnay, P., Topilko, P., and Santos, R. (2015). Prss56, a novel marker of adult neurogenesis in the mouse brain. Brain Struct. Funct. 221, 4411-4427. doi: 10.1007/s00429-015-1171-z

Kokoeva, M. V., Yin, H., and Flier, J. S. (2005). Neurogenesis in the hypothalamus of adult mice: potential role in energy balance. Science 310, 679-683. doi: $10.1126 /$ science. 1115360

Kriegstein, A., and Alvarez-Buylla, A. (2009). The glial nature of embryonic and adult neural stem cells. Annu. Rev. Neurosci. 32, 149-184. doi: 10.1146/annurev.neuro.051508.135600

Langlet, F., Levin, B. E., Luquet, S., Mazzone, M., Messina, A., DunnMeynell, A. A., et al. (2013). Tanycytic VEGF-A boosts blood-hypothalamus barrier plasticity and access of metabolic signals to the arcuate nucleus in response to fasting. Cell Metab. 17, 607-617. doi: 10.1016/j.cmet.2013. 03.004

Lee, D. A., Bedont, J. L., Pak, T., Wang, H., Song, J., Miranda-Angulo, A., et al. (2012). Tanycytes of the hypothalamic median eminence form a diet-responsive neurogenic niche. Nat. Neurosci. 15, 700-702. doi: 10.1038/nn.3079

Lee, D. A., and Blackshaw, S. (2012). Functional implications of hypothalamic neurogenesis in the adult mammalian brain. Int. J. Dev. Neurosci. 30, 615-621. doi: 10.1016/j.ijdevneu.2012.07.003

Lee, D. A., Yoo, S., Pak, T., Salvatierra, J., Velarde, E., Aja, S., et al. (2014). Dietary and sex-specific factors regulate hypothalamic neurogenesis in young adult mice. Front. Neurosci. 8:157. doi: 10.3389/fnins.2014.00157

Li, J., Tang, Y., and Cai, D. (2012). IKKbeta/NF-kappaB disrupts adult hypothalamic neural stem cells to mediate a neurodegenerative mechanism of dietary obesity and pre-diabetes. Nat. Cell Biol. 14, 999-1012. doi: $10.1038 / \mathrm{ncb} 2562$
Lledo, P. M., Alonso, M., and Grubb, M. S. (2006). Adult neurogenesis and functional plasticity in neuronal circuits. Nat. Rev. Neurosci. 7, 179-193. doi: $10.1038 /$ nrn 1867

Ma, M. S., Brouwer, N., Wesseling, E., Raj, D., van der Want, J., Boddeke, E., et al. (2015). Multipotent stem cell factor UGS148 is a marker for tanycytes in the adult hypothalamus. Mol. Cell. Neurosci. 65, 21-30. doi: 10.1016/j.mcn.2015.02.002

Matsuzaki, K., Katakura, M., Hara, T., Li, G., Hashimoto, M., and Shido, O. (2009). Proliferation of neuronal progenitor cells and neuronal differentiation in the hypothalamus are enhanced in heat-acclimated rats. Pflugers Arch. 458, 661-673. doi: 10.1007/s00424-009-0654-2

Migaud, M., Batailler, M., Segura, S., Duittoz, A., Franceschini, I., and Pillon, D. (2010). Emerging new sites for adult neurogenesis in the mammalian brain: a comparative study between the hypothalamus and the classical neurogenic zones. Eur. J. Neurosci. 32, 2042-2052. doi: 10.1111/j.1460-9568.2010.07521.x

Moraes, J. C., Coope, A., Morari, J., Cintra, D. E., Roman, E. A., Pauli, J. R., et al. (2009). High-fat diet induces apoptosis of hypothalamic neurons. PLoS ONE 4:e5045. doi: 10.1371/journal.pone.0005045

Nascimento, L. F., Souza, G. F., Morari, J., Barbosa, G. O., Solon, C., Moura, R. F., et al. (2016). n-3 fatty acids induce neurogenesis of predominantly POMC-expressing cells in the hypothalamus. Diabetes 65, 673-686. doi: 10.2337/db15-0008

Niwa, A., Nishibori, M., Hamasaki, S., Kobori, T., Liu, K., Wake, H., et al. (2016). Voluntary exercise induces neurogenesis in the hypothalamus and ependymal lining of the third ventricle. Brain Struct. Funct. 221, 1653-1666. doi: 10.1007/s00429-015-0995-x

Orellana, J. A., Saez, P. J., Cortés-Campos, C., Elizondo, R. J., Shoji, K. F., Contreras-Duarte, S., et al. (2012). Glucose increases intracellular free $\mathrm{Ca}(2+)$ in tanycytes via ATP released through connexin 43 hemichannels. Glia 60, 53-68. doi: 10.1002/glia.21246

Pencea, V., Bingaman, K. D., Wiegand, S. J., and Luskin, M. B. (2001). Infusion of brain-derived neurotrophic factor into the lateral ventricle of the adult rat leads to new neurons in the parenchyma of the striatum, septum, thalamus, and hypothalamus. J. Neurosci. 21, 6706-6717.

Pérez-Martín, M., Cifuentes, M., Grondona, J. M., López-Avalos, M. D., Gómez-Pinedo, U., Garcia-Verdugo, J. M., et al. (2010). IGF-I stimulates neurogenesis in the hypothalamus of adult rats. Eur. J. Neurosci. 31, 1533-1548. doi: $10.1111 /$ j.1460-9568.2010.07220.x

Pierce, A. A., and Xu, A. W. (2010). De novo neurogenesis in adult hypothalamus as a compensatory mechanism to regulate energy balance. J. Neurosci. 30, 723-730. doi: 10.1523/JNEUROSCI.2479-09.2010

Platel, J. C., Gordon, V., Heintz, T., and Bordey, A. (2009). GFAP-GFP neural progenitors are antigenically homogeneous and anchored in their enclosed mosaic niche. Glia 57, 66-78. doi: 10.1002/glia.20735

Robins, S. C., Stewart, I., McNay, D. E., Taylor, V., Giachino, C., Goetz, M., et al. (2013). $\alpha$-Tanycytes of the adult hypothalamic third ventricle include distinct populations of FGF-responsive neural progenitors. Nat. Commun. 4:2049. doi: 10.1038/ncomms3049

Rodríguez, E. M., Blázquez, J. L., Pastor, F. E., Peláez, B., Peña, P., Peruzzo, B., et al. (2005). Hypothalamic tanycytes: a key component of brain-endocrine interaction. Int. Rev. Cytol. 247, 89-164. doi: 10.1016/S0074-7696(05)47003-5

Schwartz, M. W., Woods, S. C., Porte, D. Jr., Seeley, R. J., and Baskin, D. G. (2000). Central nervous system control of food intake. Nature 404, 661-671. doi: $10.1038 / 35007534$

Xu, Y., Tamamaki, N., Noda, T., Kimura, K., Itokazu, Y., Matsumoto, N., et al. (2005). Neurogenesis in the ependymal layer of the adult rat 3rd ventricle. Exp. Neurol. 192, 251-264. doi: 10.1016/j.expneurol.2004.12.021

Conflict of Interest Statement: The authors declare that the research was conducted in the absence of any commercial or financial relationships that could be construed as a potential conflict of interest.

Copyright () 2017 Recabal, Caprile and García-Robles. This is an open-access article distributed under the terms of the Creative Commons Attribution License (CC BY). The use, distribution or reproduction in other forums is permitted, provided the original author(s) or licensor are credited and that the original publication in this journal is cited, in accordance with accepted academic practice. No use, distribution or reproduction is permitted which does not comply with these terms. 